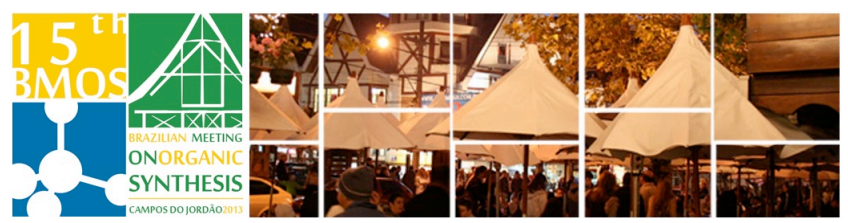

\title{
Binding site elucidation of a small molecule inhibitor of FtsZ
}

\author{
Gabriella M. Nepomuceno, David E. Anderson, Clarissa Tadeus, Qian Wang, Phillip \\ P. Painter, Katie M. Chan, Terrence E. O’Brien, Francisco J. Sarabia, Dean J. \\ Tantillo, ${ }^{*}$ James B. Ames, * Carlito Lebrilla, * Jared T. Shaw*
}

gnepomuc@ucdavis.edu

*e-mail corresponding author: jtshaw@ucdavis.edu

Keywords: FtsZ, antibiotic, cross-linking

\section{INTRODUCTION}

Bacterial infections are becoming increasingly resistant to available antibiotics, ${ }^{1}$ often through a common mechanism of evolution to prevent the binding of drug molecules. While the development of new antibiotics for established targets is one approach to combat these infections, there is a rising need to consider alternate proteins or pathways. The bacterial cell division protein, FtsZ, therefore offers a new area for therapeutic research. Recently, ${ }^{2}$ several reported Fts Z inhibitors that led to a filamentatous phenotype were demonstrated to act through non-specific interactions. Zantrin Z3 (Z3) was the only molecule to maintain activity against $B$. subtilis (BsFtsZ) and E. coli (EcFtsZ). However, limited crystal data for BsFtsZ and EcFtsZ makes the development of more potent analogs difficult.

\section{RESULTS AND DISCUSSION}

In order to elucidate the interactions between FtsZ and Z3, a thorough structure activity relationship (SAR) must be developed. Directed SAR studies, however, are challenging because the binding site is unknown and there is no co-crystal structure of an inhibitor with FtsZ. Previous work by the Shaw group $^{2}$ identified three areas of the benzoquinazoline core (Figure 1) where modifications could be made to improve potency and ligand efficiency.

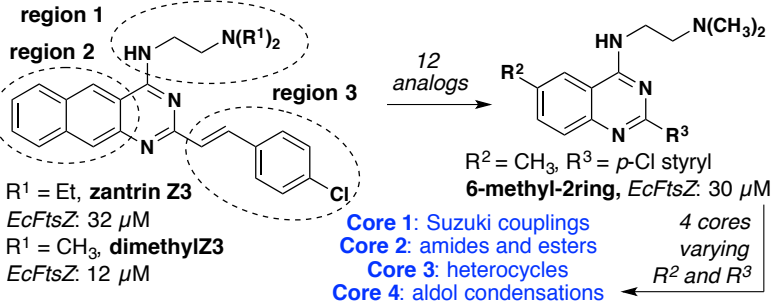

Figure 1. Early SAR in regions 1 and 2 led to improved potency and ligand efficiency.

Various 2-aminobenzoic acids were used as starting points for four cores (Figure 2) that have led to more than 30 analogs of Z3. The haloquinazoline core has been used in Suzuki couplings to introduce heterocycles via aryl boronic acids. Alkyl Suzuki substrates are also under investigation. Additionally, a Sonogashira coupling is undergoing optimization in order to access substituted triazoles. Many alkyl and aromatic amides have been tested as bioisosteres for the styryl group in Z3. However, current inhibition data suggests contracting the overall length of $Z 3$ by removing the alkene reduces its activity. Thus, aldol condensation of aromatic and alkyl aldehydes with different substitution patterns is being explored.

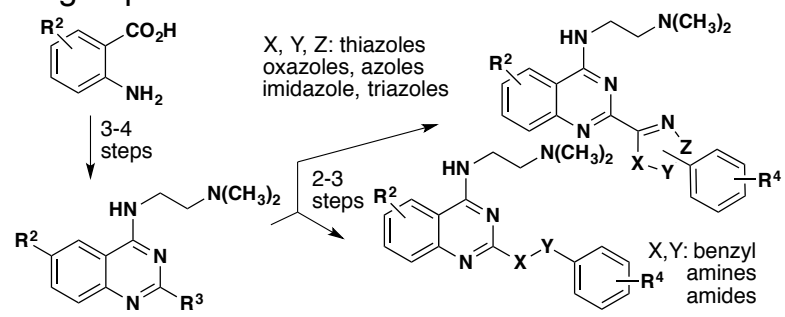

Figure 2. Synthetic sequence to core replacements of $Z 3$. $\mathrm{R}^{2}=\mathrm{Br}$ or $\mathrm{CH}_{3} ; \mathrm{R}^{3}=\mathrm{Cl}, \mathrm{CH}_{3}, \mathrm{CO}_{2} \mathrm{H} ; \mathrm{R}^{4}=\mathrm{CN}, \mathrm{OR}, \mathrm{R}$, halogens or other heterocycles.

Zantrin Z3 was further modified to incorporate a photoreactive azide for cross-linking studies. We hypothesize our docking model can be validated by mass spectrometry of the covalently bound proteininhibitor complex. With the binding site identified, computational design of new analogs will guide our SAR efforts towards a more potent drug.

\section{CONCLUSION}

Our developing SAR, computations and structural studies through mass spectrometry will contribute to a better understanding of FtsZ inhibition. As a result, our strategy for future analogs can be directed towards a particular binding site; improved specificity and activity will eventually lead to an antibiotic with a novel mode of action.

\section{ACKNOWLEDGEMENTS}

I thank the Shaw, Tantillo, Ames and Lebrilla groups for their insightful discussions and the NIH (R01 AI08093), the US Department of Education for a GAANN fellowship, and the Alfred P. Sloan Foundation Minority PhD Scholarship.

\section{REFERENCES}

Walsh, C. Nature 2000, 406, 775-781

2 Anderson, D. E.; Kim, M. B.; Moore, J. T.; O'Brien, T. E.; Sorto, N. A. Grove, C. I.; Lackner, L. L.; Ames, J. B.; Shaw, J. T. ACS Chem. Biol. 2012, 7, 1918-1928.

Omega2; Openeye Scientific Software, Inc., Santa Fe, NM, USA www.eyesopen.com; 2009. FRED 2.2.5ed.; Openeye Scientific Software, Inc., Santa Fe, NM, USA. www.eyesopen.com; 2009. 